\title{
Investigating Ethical Design Requirements for Digitalized Healthcare Support: The Case of Ambulatory Physiotherapeutic Assistance Systems
}

\author{
Marius Müller \\ University of Siegen \\ marius.mueller@uni-siegen.de
}

\author{
Oliver Heger \\ University of Siegen \\ oliver.heger@uni-siegen.de
}

\author{
Björn Niehaves \\ University of Siegen \\ bjoern.niehaves@uni-siegen.de
}

\begin{abstract}
Due to the advent of digitalized healthcare services and de-centralized structures, the tele-medical support of therapeutic treatments is increasingly in the focus of researchers and practitioners. Here, systems offering an interface between patients and physicians emerge as a fruitful way to reduce clinical visits and, thus, increase patient satisfaction and health. Yet, research on requirements for such systems has largely focused on patients who are not able to fully grasp the issues associated with such technologies due to their novelty and the changes they entail. With this study, inspired by the Responsible Innovation framework, we investigate the case of an ambulatory physiotherapeutic assistance system. We conducted four focus group workshops involving experts from different domains in order to integrate multiple stakeholder perspectives and thereby explore system design requirements. Our findings indicate that patient autonomy, security, privacy, competence and socio-cultural aspects contain relevant technological implications, each involving multiple design requirements.
\end{abstract}

\section{Introduction}

Healthcare is increasingly supported by digital solutions, especially to assist medical laypersons in the implementation of therapeutic procedures. The medical informatics domain is gaining more and more attention since around the turn of the millennium. This scientific field puts effort into the examination of the effectiveness of medical systems and their potential to support therapeutic outcomes $[9,15]$. Particularly the design of therapeutic assistance systems for medical laypersons raise questions about its ethical implications. Whereas traditional applications, such as an ERP system or an online communication tool, do not entail major threats to the physical well-being of its user, systems that assist therapists or patients in performing therapeutic activities can potentially cause serious harm to the user. For instance, immature technology functionalities or improper visual guidance can lead to deterioration of the therapy and physiological injuries [34]. This, in turn, can lead to uncertainties, a lowered self-efficacy or even mental stress since the own health is affected [26], overshadowed by the omnipresent risk of data leakage and privacy issues [1]. In addition to the question whether a system being potentially harmful for health is ethically acceptable, individuals have reservations when it comes to using it, which may lead to resistance against or rejection of the technology [28].

One special characteristic of the class of ambulatory therapeutic assistance technology is the area of tension involving different stakeholders and interests as well as new spatial and temporal settings. This calls for new investigations, especially from an ethical perspective addressing the different parties involved. The design of systems being implemented in unconventional settings (e.g. at home) in order to aid the patients' treatment routines comes along with two major issues: (1) Due to the systems' novelty and the explosiveness of their purpose (i.e. patients' health and quality of life), most patients cannot rely on experiences when it comes to assessing design requirements and technical features. (2) Designing, developing and evaluating such a system incorporates a highly heterogeneous structure of stakeholders, not only within the group of patients but also within the groups of developers, physicians and therapists, scientists, health insurances, relatives and more. Their insights are able to inform a knowledge base for future research and contribute to a multifaceted examination.

With regard to these issues, the field of Responsible Innovation (RI) provides a promising framework for integrating ethical implications of technology into the design process. The objective of RI is the ethical acceptability, sustainability, and societal desirability of research and innovation [37]. It calls for the involvement of stakeholders in the design process as well as the orientation towards normative anchor points such as human health [37]. It furthermore demands anticipative activities in the design process to analyze intentional and unintentional consequences of an 
innovation for identifying ethical problems as early as possible [25]. In order to explore the ethical design of therapeutic assistance systems and taking RI into account, we aim to anticipate ethical implications of the assistance system from three different expert perspectives (developer, scientist, physician) as a starting point of our design process and, from that, to derive suitable design requirements. The reason we focus on expert perspectives as a first step is that their field and technical expertise allows us to begin with exploring, comprehending and anticipating realistic technological implementations before confronting patients with reasonable scenarios, benefits and harms. Hence, our study is guided by the following research questions (RQs):

RQ1: Which ethical implications of a therapeutic assistance system can be anticipated from relevant expert stakeholder perspectives?

RQ2: Which design requirements can be derived from these ethical implications?

\section{Related Work}

\subsection{Assistance Systems in Healthcare}

Research on medical assistance systems with a focus on therapeutic support is rather scarce in the IS domain. To date, much research has been conducted investigating tele-medical systems, which are able to interface patients and physicians, fostering a decentralized healthcare structure. Here, parallels between the domains of medical informatics, Human Computer Interaction (HCI) and IS can be detected, for instance with regard to the development and design of healthcare systems [15]. One major research stream deals with the development of electronic health records, enabling physiotherapists to store patient data in a mandatory structured manner and share the data with other healthcare providers of the patient [8]. Implementing such information systems makes vital information accessible for the patients and, thus, serve as an interface between clinical and home environments [15]. In this vein, Chae et al. [9] conducted a study on the effectiveness of tele-medical systems. Major findings indicate that they can lead to less clinical visits and higher patient satisfaction regarding the medical treatment, fostering an economic and efficient way of healthcare support. Complementary, Alaiad and Zhou [2] empirically investigated factors constituting the user adoption of smart home healthcare systems promoting a ubiquitous health support. Their results indicate that expected effort and life quality as well as social influence affect the patient's intention to use such a system. Research largely unveiled important factors influencing the technological effectiveness and the adoption by the user.

Nevertheless, with specific regard to the development of physiotherapeutic assistance systems, Haux [18] identified several challenges that need to be addressed by researchers in the future. A major challenge is constituted by the digitalized therapeutic interventions, which should come along with as little strain on the patient as possible. Additionally, providing crucial and extensive information to the patient as well as enabling rigor documentation of the therapy process - involving multiple applications and exercises performed by the patient - and associated knowledge represent important issues that need to be tackled. With these challenges in mind, looking at digitalized therapy support from an ethical point of view seems promising, since the individual needs, expectations, and concerns of the patient play a major role in the design process.

To that end, multiple studies on ethical challenges and issues in the design process of ambulatory healthcare systems have been conducted. For instance, with regard to mHealth technologies, the importance of user feedback and the accompanying subjectivity due to individual value-systems and preferences has been shown [5]. Within the context of stay-at-home patients and their relations to caregivers, empiricism unveiled interpersonal tensions, which can occur in the home setting due to counteracting values, needs and expectations [7]. Apparently, many studies in the context of ambulatory therapy assistance focus on empirical and evaluative investigations with the patient as the unit of analysis. Since the case at hand incorporates an area of tension, which involves many actors (patients, caregivers, physicians, etc.) and settings (home, clinic, practice), the matter calls for supplementary methods aiming at a more holistic view, which is able to surpass tensions caused by uncertainty and subjective points of view. In order to address the ethical aspects of system design systematically and deliberatively, the Responsible Innovation approach represents a suitable theoretical and methodological lens, since it explicitly integrates perspectives of relevant stakeholders.

\subsection{Responsible Innovation}

RI assumes that technology is not only technically but also socially and politically constituted and therefore suggests that scientists, funders, innovators, and others share a collective political responsibility [33]. RI calls for a "transparent, interactive process by which societal actors and innovators become mutually responsive to each other with a view to the (ethical) acceptability, sustainability and societal desirability of the innovation process and its marketable products" [36] (p. 9). Within 
the RI framework, the anticipatory dimension requires methodologies that enable foresight to surface issues and explore impacts that may otherwise remain uncovered [25]. RI is applied across many different engineering and social sciences including the fields of IS and healthcare. In a study on ethical implications of emerging technology, innovative technological features such as "detailed understanding of the user", "autonomy", or "power over the user" have shown to be very significant from an ethical point of view and relate to ethical issues such as privacy, trust, liabilities, and digital divides [31]. The IS community is therefore called upon to engage more in ethical discourses around new technology [32]. Based on a demand for more critical research in IS, Stahl et al. [30] argue that focus groups as a method of data collection can contribute to the field of IS. With regard to healthcare, RI calls for proactive actions by a variety of stakeholders to address ethical and social implications of new technologies in healthcare to shape the innovation landscape [10]. Based on the findings of three focus groups comprising, inter alia, hospital managers, industrial designers, and medical device manufacturers, a study identified a variety of health care system challenges that technological innovation could help address [12]. The study states that anticipation needs to pay attention to the varying contexts of use in health organizations and home. Developers need to remain open to the views of others, and to be responsive to new knowledge and values, proving the applicability of the method within our study.

\section{Methodological Approach}

\subsection{Research Design}

Case study research. The context of our study is highly explorative and context-specific. Since little research has been done on the class of assistance systems under investigation, a case study research design investigating the dynamic setting at hand was deemed valuable and promising [13]. We aim for insights on context-related system design within a transdisciplinary spectrum of perspectives, needs, requirements and expectations. Hence, our case involves experts from different domains.

Focus group approach. For the explorative purpose of our case study, we conducted a qualitative focus group approach. The aim of a focus group investigation is to assemble a group of chosen individuals and collect data through group interaction and discussion on a given topic from personal experience [22, 24, 30]. Focus groups as a qualitative research method are highly underutilized in the IS domain [24, 30]. Nevertheless, they exhibit multiple strengths and advantages. According to O'hEocha et al. [24], focus groups direct attention to a specific topic just like interviews, but also facilitate discussion and, hence, emergence of consensus as well as conflicts. Furthermore, within a short period, researchers are able to collect rich data such as "attitudes, feelings, beliefs, experiences, and reactions in a way that is not feasible using other field methods" [30] (p. 4). Group discussion helps in increasing depth of data due to the surfacing of opinions, ideas and concerns that would otherwise not be externalized. Interactions can fill knowledge gaps and facilitate a better understanding through stimulating idea association and recall, helping the participants to quickly arrive at a common understanding of the given topic [15, 30]. Hence, focus groups are suitable for investigating unexplored and emerging topics such as assistance systems design for therapeutic and health purposes, since this methodology is already established in the healthcare domain $[15,21]$.

Case description and usage scenario. A heterogeneous, multidisciplinary group with differing values, means, judgments and opinions constitutes the sample participating in the study. All of the participants take part in a national research project on the development of a therapeutic assistance system and are experts in their respective field. The project representing our case deals with the demonstrative development of a mobile system, which is capable of assisting physiotherapeutic treatments, particularly the Vojta therapy. This therapy can give access to elementary movement patterns of patients with an impaired central nervous system by applying a stimulus on the patient's body, which evokes automated movements. Here, three initial usage scenarios represent the baseline for further investigations. First, the patients' caregivers are able to utilize the system and receive visual and contextual aid in performing treatments with the patient, for instance, in case of severe immobilization of the patient. The ambulatory character of the system enables them to be spatially independent, facilitating a smaller amount of clinical visits and less face-to-face time with the therapist. Second, the system can work as a control tool, capturing and analyzing the therapeutic movements. The therapist or physician can utilize the data to optimize the therapeutic process, which can lead to quicker and possibly stronger results regarding the patients' health and the effectiveness of treatments by laypersons. Third, the system can serve as a training tool within the education of future therapists by providing fundamental teaching content and treatment guidance.

Currently, the system assembles a combination of software modules, such as user interface and movement analysis algorithms, as well as hardware components. The latter cover depth cameras, body sensors, and 
pressure plates that are able to measure the patient's movements in a non-clinical environment, for instance, at home. The aforementioned usage scenarios and the technical set-up form the workshops' basis for discussion.

\subsection{Data Collection and Analysis}

We carried out four moderated focus group sessions engaging a sample of 19 experts. To organize the sessions and allow theoretically driven concepts to emerge, we followed a two-step approach consisting of an initial (focus group 1) and subsequent data collection phase (focus groups 2 to 4 ), paired with iterative data analysis. The first session aimed to explore ethical implications of the system development open-mindedly, thus, addressing RQ1. The three subsequent sessions cover investigations on system requirements based on the initial findings, answering RQ2.

The initial session, engaging 19 participants, was a heterogeneous "full group" workshop [17] involving multiple disciplines, each fulfilling different roles in the development process, i.e. medicine, technical development, IT consulting and social and computer sciences. The participants were about equally distributed across the disciplines. The session took 120 minutes. The workshop was executed in four phases: (1) The moderator gave an introduction on ethical issues related to technology design in order to sensitize the participants for topics beyond "traditional" ones such as security. (2) The three usage scenarios and the technical status-quo was provided to achieve a common starting point. (3) Each participant was asked to write down up to five ethical issues and design challenges that come to mind. (4) The individual results were presented and discussed in plenary. This way, we achieved an interdisciplinary discussion encouraging the experts to think beyond their disciplines. The results were captured in the form of written notes being attached to a whiteboard and categorized by content related similarities. For instance, one category dealt with privacy and data security issues that are inherent in the requirement engineering phase of a health support system dealing with highly personal data such as biosignals and physical markers (e.g. blood pressure, heart frequency or oxygen level). After the workshop, a set of major themes was derived from the data within a first data analysis iteration (see Table 1). The theme construction was done by iteratively clustering the notes from the first workshop in order to identify underlying requirements representing the gathered ethical aspects of the system design.

Ethical issues with regard to patient intimacy and privacy as well as security issues such as effective data loss prevention and an appropriate access control to sensible data (e.g. diagnosed disease(s), medication, therapy progress) form the theme of Privacy and Security (T1). The Autonomy theme (T2) involves issues with regard to the extent of control a user has during the therapeutic application, the freedom of choice in going alternative routes (e.g. more convenient, less hurtful treatments) as well as the trustworthiness of the system assistance functionality. Competence (T3) refers to the development of therapeutic skills regarding the treatment and the prevention of competence reduction due to a high amount of digital assistance. The latter hereby can be caused by increasing user dependence on system support, exploiting the user's natural learning curve. The fourth theme Design for All (T4) deals with socio-cultural and demographic aspects of the system design. These involve issues such as proper handling of the system by the elderly or people with less computer knowledge as well as categorically excluding other groups from using the system due to certain characteristics (such as disembodiment, poverty or skin color).

Table 1. Emerging themes

\begin{tabular}{|l|l|}
\hline Theme & Definition (self-phrased) \\
\hline $\begin{array}{l}\text { T1: Privacy \& } \\
\text { Security }\end{array}$ & $\begin{array}{l}\text { The degree to which the system } \\
\text { safeguards the user's privacy and } \\
\text { protects data from unauthorized access. }\end{array}$ \\
\hline T2: Autonomy & $\begin{array}{l}\text { The degree to which the user is able to } \\
\text { autonomously control the system } \\
\text { behavior during a therapy session. }\end{array}$ \\
\hline $\begin{array}{l}\text { T3: } \\
\text { Competence }\end{array}$ & $\begin{array}{l}\text { The degree to which the system } \\
\text { utilization preserves or increases the } \\
\text { individual competence of the user. }\end{array}$ \\
\hline $\begin{array}{l}\text { T4: Design for } \\
\text { All }\end{array}$ & $\begin{array}{l}\text { The degree to which the system is } \\
\text { designed for a heterogeneous } \\
\text { population (e.g. age, gender, culture). }\end{array}$ \\
\hline
\end{tabular}

Subsequently, we enriched the set of themes with literature [27]. This served the purpose of (1) gaining a deeper understanding of the respective phenomenon and (2) achieving a higher degree of bibliographic connectivity. Since the uncovered themes have already been examined within other contexts, we utilized this knowledge and included it in the following focus group sessions guiding the moderated discussions as additional input. By this, we were able to foster the major themes and gain insights on how they behave in the specific context of ambulatory physiotherapeutic assistance systems. Table 2 shows the theoretical integration of our interim results.

Table 2. Theoretical integration of themes

\begin{tabular}{|c|l|}
\hline Theme & Theoretical Link(s) \\
\hline T1 & $\begin{array}{l}\text { Threat avoidance [22]; Privacy calculus [19]; } \\
\text { Control over personal data [6] }\end{array}$ \\
\hline T2 & $\begin{array}{l}\text { Perceived Behavioral Control, Computer anxiety } \\
{[14]}\end{array}$ \\
\hline
\end{tabular}




\begin{tabular}{|c|l|}
\hline T3 & $\begin{array}{l}\text { Job effectiveness and performance changes [16]; } \\
\text { Dependency and extent of use [20] }\end{array}$ \\
\hline T4 & Digital Divide [23] \\
\hline
\end{tabular}

Following the initial data analysis and its theoretical integration, we designed three subsequent focus group sessions based on the initially gained insights. We followed a "mini group" design [17], for which each workshop took about 90 minutes and involved a small, homogenous group belonging to a dedicated discipline (see Table 3), exhibiting a shared space of meaning and understanding. Hereby, we aim to gain detailed insights from specific perspectives, enriching our data across disciplines and antagonizing narrow, possibly biased statements.

Table 3. Focus group constellations

\begin{tabular}{|c|l|c|}
\hline Session & Discipline & $\#$ \\
\hline FG1 & Heterogeneous (see above) & 19 \\
\hline FG2 & Technical development and consulting & 7 \\
\hline FG3 & Research (computer and social sciences) & 6 \\
\hline FG4 & Healthcare (physicians and therapists) & 6 \\
\hline
\end{tabular}

Each of the three workshops consisted of four cycles covering the emerged themes. Each theme (T1 to T4) was handled separately. Here, one cycle dealing with one theme consisted of (1) theme definition and refinement, (2) requirement derivation and definition by each participant, (3) collective sighting of requirements, identifying parallels and conflicts, and (4) exploring possible technological implementations. The order of themes during the workshops was randomized in order to antagonize possible negative effects such as fatigue during the sessions. We integrated the results and insights from a workshop within the subsequent ones, fostering a theory-driven emergence of data. This structured format exhibits a high moderator input [24], leading to comparable and consistent, yet context-bound results. Data was collected in the form of digital audio recordings and ensuing transcription, written in vivo protocols prepared by one of the authors as well as index cards written by the individual participants. This led to three distinct (i.e. one per workshop) sets of design requirements addressing the formerly identified themes.

Analyzing the data was performed in two deductive steps: (1) Each cycle within a single workshop session (representing one particular theme) was analyzed separately, leading to a specific set of subthemes. (2) Afterwards, we looked for correlations, similarities and occurring dissonances between these identified subthemes and those from the other two workshops, leading to an aggregated list of subthemes for each of the four themes $\mathrm{T} 1$ to $\mathrm{T} 4$ across disciplines.

\section{Findings}

In the following, we present the four emerged themes, each involving several subthemes, representing concrete requirements, and thus relating to RQ2. The quotations given below were translated into English analogously with minor adjustments regarding grammar and syntax and preserving the statement's meaning. Each statement represents the consensus within the respective group.

\subsection{Privacy and Security}

Openness and Connectivity. In order to foster a high degree of user privacy, security of personal data as well as intimacy, according to the professionals from the medical domain, the system can be treated as a closed environment, comparable to a traditional patient record. "Patient data is filed in a medical record, which is closed [for others]. We would handle this data like every other information, for instance, like EKG pictures. We would not make any exceptions here. It would be a closed system, which would make an application-based system difficult. The data must not land in any cloud. The system should not have an internet connection at all. [...] Removing the data from the building [the clinic] is not allowed." (FG4) Furthermore, the responsibility over the data lies with the individual patient, for instance, "the patients may take their $x$-ray images home with them at any time." (FG4)

Data Storage. This subtheme deals with how the system can handle different incurring forms of measurement data. A developer suggested to withdraw raw material such as video data which shows the patient and instead analyze these files on the fly. "We should avoid storing videos and pictures of the patient. Which means I analyze the incurring data, look at the results, and withdraw the original data. Basically, the need for this data is no longer existent. [...] Let us say you get a feedback in the form of green, yellow, and red signals, the treatment was okay or not okay. I would only use the video data to capture the movements. [...] We should delete this data once we did our analysis." (FG2) This requirement lies in the vein of data economy, only storing highly abstract measurement data (e.g. frequency of movements) that cannot be attributed to a specific person. "The movements and the accuracy can be analyzed and visualized, but pictures of the actual body are not saved." (FG3)

Simulation. As an alternative to the subtheme above, the simulation of movement data which uses an abstract, humanoid model emerged as a way of storing (originally) personalized data. Since, for instance, video material can be useful later on when " [...] looking at the measurement data and comparing it to what actually 
happened in the video" (FG2), simulation can depersonalize data: "In case the patient does not want to capture a video, you can simulate the movement information using some human or skeletal model. Others will see a mapped model hiding the patient's identity. Another option might be to blur or hide some body portions like the face, which are not essential to capturing the movements." (FG3)

Transparency. This subtheme covers the system feature of achieving awareness of the patient towards the ongoing technological processes such as data capturing, analyzing, and storing. A computer scientist suggested that "the system might show some notifications on the user interface, such as what the software algorithm is currently capturing and evaluating. [...] So the user gets feedback on what the system is doing right now and what kind of data incurs." (FG3) The users could dynamically negotiate whether they want to produce specific sets of data in order to preserve their individual privacy. With regard to this, a technical developer mentioned the capability of turning system features on and off manually and autonomously: "The user should be able to turn every feature on and off, for instance, the storage and transfer of incurring data. You [the user] should be able to configure different scenarios within the system with regard to data security. Do you want to store video data? If yes, shall it be stored centrally?" (FG2) In contradiction to the "closed system" mentioned above, the open nature here does not tackle unauthorized access by externals but the transparent and flexible implementation of system features.

\subsection{Autonomy}

Voluntary Use. With regard to user autonomy, the participants from the medical domain attached importance to the voluntariness of the system usage itself. On this general level a therapist stated: "If you want to be captured with regard to your movements, you turn it [the system] on and vice versa. Easy decision. You do not have to use it. There are no disadvantages in doing so. However, there are some advantages such as comparing your handles to the ideal ones and, thus, increasing the treatment quality at home and feeling more confident. You can consider it a supportive offer, but not mandatory." (FG4) This defines the purpose of the system, which is complementary and less restraining.

Individualization. In order to retain the users' potentials regarding the treatment itself, the system should allow individual approaches and handles during a therapy session. According to a developer, this can be done by initially setting up specific goals of the therapy session. "You can ask the user. What do we want to achieve? So you can tell the system that you want to accomplish this by your own and in case you are wrong, the system can inform you automatically or by user inquiry. [...] Predefining the exact procedure lowers the potential. People will say 'that is wrong, I would rather proceed like this'." (FG2)

Data Interpretability. Enabling individual interpretations of captured data during the therapy session emerged as a subtheme. A physician mentioned the pulse oximeter (a clip attached to the patient's finger measuring the oxygen saturation of the blood) as a representative example: "The range [of the saturation] is from 0 to $100 \%$, values under $90 \%$ are naturally bad, so the oximeter gives a signal when the value drops lower. However, in case of premature babies, values like $91 \%$ are normal and it can drop to $86 \%$ sometimes. In this case, there is no health risk, so the parents adjust the alarm limit to $85 \%$. [...] You get a feeling for how to adapt the alarm to the real situation." (FG4) It becomes evident that the interpretation of the system outputs lies with the user. "The system cannot make decisions regarding the real meaning of the measurement results. [...] Only the doctor can do that, looking at the individual patient's situation. [...] In turn, this can lead to more user independence and confidence." (FG4) This degree of freedom however can affect the therapy progress, thus, suitable control mechanisms need to be implemented.

Access Authority. With regard to the extent of access certain user groups have to the system settings and configurations, this subtheme suggests to look at different levels of authority. The participants mentioned implementing both a detailed and broader access as well as a rather limited one. The former addresses therapists and physicians, being able to configure individual option sets for the patients, leading to the latter access mode. "The therapist should have a more generic level of access to the software settings, whereas the patients have a more limited access because they are not that versed in medical issues." (FG3) Furthermore, the expert, after performing an initial, user-individual setup, can adjust the system periodically in collaboration with the patient, which in turn can positively affect the patient's trust in the operating principles of the technology. "The system should be adjusted within the clinic for the first time. [...] In the first meeting, the therapist and the patient can choose the features together. [...] In the next meeting, they can adjust the settings in case the patient feels uncomfortable with the way the system works." (FG3) This comes along with a certain degree of visibility with regard to the available options and features, lowering a potential information overload and simplifying system handling: "You do not need to show the user all available options and features of the system." (FG3) 


\subsection{Competence}

Accuracy. During the different cycles exploring the competence theme, the accuracy issue emerged as a major subtheme across all disciplines. On the one hand, the experts from the medical domain demand a very high degree of accuracy when it comes to measuring, documenting and analyzing movements during a treatment session. For instance, one therapist stated that "[...] it is an absolute mandatory criterion having a high [movement] detection rate of $99 \%$ and above so that it makes any sense. Otherwise, it is just a gimmick." (FG4) On the other hand, a computer scientist working on the pattern recognition algorithm (FG3) mentioned that an accuracy level of 85 to $90 \%$ would be a satisfactory and targetable goal from a technical point of view. Here, according to a physician, this comparably low accuracy does not satisfy the proper digitalization of an established therapeutic treatment model, since "we have a very complex diagnostic and treatment model, which we developed over decades and is working very well with regard to the patient care. If you are measuring superficially [...] and cannot represent the complexity [...] you may have something very plausible [and efficient] but do not satisfy the diagnostic and therapeutic possibilities." (FG4) The system can be "fascinating and efficient" but rather inaccurate. Thus, it still requires the profound competence of an expert.

Level of Expertise. Another major subtheme discussed throughout the workshops is constituted by the different degrees of competence and knowledge the user groups (i.e. physicians, therapists, patients and caregivers) have. Whereas newcomers, apprentices and laypersons lack certain skills regarding the therapeutic treatment, experts often have huge pools of knowledge at their disposal and, throughout the years, have developed individual treatment procedures beyond the textbooks. One physician for instance claimed a high degree of adaptability: "Our novices use a copied note when they are on the station, because they cannot memorize the content of the examination, yet. We, the experienced ones, already stored this sheet on our internal hard drive, so I perform only the steps that I need. [...] The digital [examination] catalogue wants to be processed, so does it take away my variability [and flexibility]?" (FG4) According to many participants, this issue can be tackled through the implementation of different modes, i.e. a training mode providing detailed information during the treatment and an expert mode, in which the system only sporadically supports the user with meaningful information without giving linear guidelines. "Within a training system, there could be a visualization you can look at, which is guiding you. In the next step [i.e. mode] the visuals are turned off and there is only a signal when doing the handles. Within the high-level version, the system is inactive and only reacts in case of treatment errors." (FG2) Alternatively, the support system could only be active periodically in order to antagonize a loss of competence: "It would be a good thing to run through the process without any kind of automatization, [...] having temporal intervals where the therapists only [treat by themselves]." (FG2)

Feedback. According to the developers and researchers, different forms of user feedback can be a promising way to maintain and build competence. To foster a certain degree of awareness, the system should provide both instant and long-term feedback. Whereas the latter rather serves a documentary purpose, instant feedback is able to efficiently support the treatment session. Here, subliminal forms of feedback stem useful. "The feedback should not consist of graphical elements. It could be a sound or a light signal, so that the person is still involved in the activity [...] and does not have to look at a monitor. This at most could be used for instruction purposes. Minimalistic visualization." (FG2) Auditory signals have the advantage of a higher reproducibility: "When I am looking at the display and want to reproduce the rhythm [of movements], I do not have a pattern the brain can memorize. In case of the sound, I am feeling it. We have to reproduce that." (FG2)

Coexistence. Several workshop participants from different domains mentioned that the system most likely could not function without any sort of manual input by a human being involved in the actual treatment. Hence, the user and the system mutually assist each other with respect to their capabilities. "We cannot say 'just take the system, apply to every patient and always get the exact results'. I think the major competence needed is to manually give certain inputs such as 'this is how it has to look like and this is the expected outcome for this particular patient'." (FG3) Hence, the system cannot substitute the expert guiding the therapeutic treatments. "We have to look on both sides. We will take the knowledge from the therapist and the data from the system. We have to make an intersection to figure out whether they make the same decisions." (FG3) A therapist states, "[...] we can help the system to classify the movement measurements and the system can assist us in capturing and documenting the movements of the patient." (FG4)

\subsection{Design for All}

Accessibility. One subtheme emerging throughout the workshop sessions deals with the design and implicit marketing concept of the system. In order to achieve a high degree of accessibility by a broad population, the participants suggest different ways of implementation. "If you design a system any user can access, for 
instance, by using his or her smartphone and a dedicated software application, you will increase the generality and the number of potential users because many people own a smartphone these days." (FG3) However, this solution comes with difficulties as well: "There is some trade-off. Imagine the scenario of an elderly person not owning a modern smartphone. For that person, we could provide some device like a tablet, running only one application with easy navigation and pairing itself automatically with the rest of the system." (FG3) A dedicated hardware device, in turn, is most likely affiliated with greater costs. Nevertheless, the financial health insurance support represents a possible route: "In case the system is very expensive but exhibits a high value with regard to the rehabilitation quality and efficiency, the public community [i.e. health insurance] will most likely pay for that." (FG4) Hence, delivering a high benefit to the healthcare system could facilitate the financing of the system and make it more accessible to the public. "If we can spread the costs over many people, using the system remains attractive for all." (FG2)

Guidance. As mentioned above, intuitive and easy to learn controls can render the system more accessible and usable for certain user groups. With regard to the necessary degree of usability, according to some participants, it can be helpful to renounce given freedom and autonomy of the user for the sake of a more streamlined guidance. "Closely related to user autonomy, in case an elderly person has difficulties using the system, the system can be configured accordingly, so the user is automatically led by the system [during the treatment]." (FG3) The same can hold true for potential users with low computer skills: "Usability should be ensured for less technologically affine people." (FG2)

Data Heterogeneity. The last subtheme is constituted by the set of various kinds of data the system generates. In order to address the beliefs, values and expectations of a broad variety of potential users with regard to defining characteristics such as culture, religion, demographics and appearance, the participants stated that the system should not rely on a single mandatory data source but on many. With regard to a camera system capturing patient movements, a computer scientists stated: "I can imagine to set up sensory features including color information and noncolor information, for instance depth information or skeletal information. There are many features [of the patient's body] the system would be able to classify, regardless for instance the patient's skin color." (FG3) Hence, the individual user could have the freedom to choose amongst different data sources without limiting system functionality.

\section{Discussion}

The findings of our study, representing the initial step within the ethically driven case of an ambulatory therapeutic assistance system, indicate several contributions for both theory and practice, motivating further investigations and (design) theory building.

Implications for theory. The paper is able to contribute to the body of knowledge regarding the ethically driven research on ambulatory therapeutic systems. Since the systems most likely change temporal and spatial settings and the way therapies are performed, our findings shed new light on already known factors. In order to achieve system adoption and acceptance, decentralized patient-generated data requires new privacy concepts such as discarding original data and simulate it generically. Second, the degree of user autonomy raises the question on how much freedom the user should have, since actions may stand in conflict with the therapist's specifications. Besides allowing individual usage scenarios, the interpretability of system outputs complements our understanding of user autonomy. Third, fostering and utilizing individual skills represents a major challenge, since an inaccurate therapeutic treatment support can lead to serious health issues. The construct of user competence, in this case, consists of both computer efficacy and therapeutic knowledge. With these theoretical implications, the findings of our study simultaneously contribute to ethical implications of new technologies in healthcare, which the field of Responsible Innovation calls for [10].

Implications for practice. Our results represent technical requirements, which developers of therapeutic healthcare technologies are able to implement within ambulatory systems aiming to interface practitioners and their patients. For instance, our results call for new ways of on-the-fly data analysis mechanisms, parallelizing treatment and examination and superseding the storage and transfer of sensible data. Here, modern pattern recognition algorithms promise efficient ways of data analysis [38]. This also addresses the given limitations regarding internet connectivity and openness of the system. Additionally, our results indicate that developers should have the coexistence of human and technology in mind. Implementing ways of mutual assistance while respecting the user's expertise and tacit knowledge represents a promising way of maintaining or even promoting individual skills and user confidence. Here, so-called exergames combining gaming elements and physiological exercises provide a promising technology, allowing the user different ways to fulfill the treatment goal [3, 29]. Subsequently, developers should consider the hardware and software configuration when designing a therapeutic assistance system. For the sake of accessibility, an application- 
based support using consumer technologies (e.g. smartphones), contrary to common expectations, can lead to excluding groups of patients in need, calling for innovative and cost-efficient visualization devices [4, 34].

Limitations. The qualitative case study approach exhibits certain weaknesses. For instance, case studies may produce insights that are very detailed yet lack a superordinate perspective. Findings are prone to be idiosyncratic and rather narrow, hampering the ability to raise the generality of the gained insights and derived concepts [13]. Additionally, the sample of the case shared membership within a project. Underlying common goals can lead to biases and suppress 'out of the box' thinking. Furthermore, there are mentionable cons of using focus groups as a data collection method. Group interactions during the session can lead to conformity of views and opinions, which are predefined by dominant characters in a group and lack reproducibility [35].

Future work. Since this study is designed as an initial step within a system design process, focusing on experts, several future research opportunities arise. First, the conduction of semi-structured interviews with patients and caregivers seems promising, illuminating complementary perspectives. This enables a BetweenMethod-Triangulation [35] of findings aiming at an integrative, domain-spanning design concept, gaining deeper and more comprehensive insights on the ethically legitimized construction of ambulatory therapeutic assistance systems. This method enables integrative conclusions, leading to the promotion of knowledge production $[11,35]$. Second, the emerging system concept enables the derivation of (normative) design hypotheses, which can be analyzed with quantitative methods (e.g. surveys or lab experiments).

Acknowledgements. This research article was supported by the SenseVojta project granted by the German Federal Ministry of Education and Research (BMBF) (No. 13GW0166E).

\section{References}

[1] M. Al Ameen, J. Liu, and K. Kwak. 2012. "Security and privacy issues in wireless sensor networks for healthcare applications". Journal of medical systems, 36(1), pp. 93-101.

[2] A. Alaiad, and L. Zhou. 2015. "Patients' Behavioral Intentions toward Using WSN Based Smart Home Healthcare Systems: An Empirical Investigation". In 48th Hawaii International Conference on System Sciences (HICSS), pp. 824-833.

[3] G. Alankus, A. Lazar, M. May, and C. Kelleher. 2010. "Towards customizable games for stroke rehabilitation". In
Proceedings of the CHI Conference on Human Factors in Computing Systems, pp. 2113-2122.

[4] S. Ananthanarayan, M. Sheh, A. Chien, H. Profita, and K. Siek. 2013. "Pt Viz: towards a wearable device for visualizing knee rehabilitation exercises". In Proceedings of the CHI Conference on Human Factors in Computing Systems, pp. 1247-1250.

[5] M. Barry, K. Doherty, J. Marcano Belisario, J. Car, C. Morrison, and G. Doherty. 2017. "mHealth for Maternal Mental Health: Everyday Wisdom in Ethical Design". In Proceedings of the CHI Conference on Human Factors in Computing Systems, pp. 2708-2756.

[6] F. Bélanger, and R. E. Crossler. 2011. "Privacy in the digital age: a review of information privacy research in information systems". MIS quarterly, 35(4), pp. 1017-1042.

[7] A. B. Berry, C. Lim, A. L. Hartzler, T. Hirsch, E. H. Wagner, E. Ludman, and J. D. Ralston. 2017. "How Values Shape Collaboration Between Patients with Multiple Chronic Conditions and Spousal Caregivers". In Proceedings of the CHI Conference on Human Factors in Computing Systems, pp. 5257-5270.

[8] R. Buyl, and M. Nyssen. 2009. "Structured electronic physiotherapy records". International journal of medical informatics, 78(7), pp. 473-481.

[9] Y. M. Chae, J. H. Lee, S. H. Ho, H. J. Kim, K. H. Jun, and J. U. Won. 2001. "Patient satisfaction with telemedicine in home health services for the elderly". International journal of medical informatics, 61(2), pp. 167-173.

[10] G. Christie. 2017. "Progressing the health agenda: responsibly innovating in health technology". Journal of Responsible Innovation, pp. 1-6.

[11] D. DeLuca, M. J. Gallivan, and N. Kock. 2008. "Furthering information systems action research: a postpositivist synthesis of four dialectics". Journal of the Association for Information Systems, 9(2), p. 48.

[12] O. Demers-Payette, P. Lehoux, and G. Daudelin. 2016. "Responsible research and innovation: a productive model for the future of medical innovation". Journal of Responsible Innovation, 3(3), pp. 188-208.

[13] K. M. Eisenhardt. 1989. "Building theories from case study research". Academy of management review, 14(4), pp. $532-550$

[14] C. Elie-Dit-Cosaque, J. Pallud, and M. Kalika. 2011. "The influence of individual, contextual, and social factors on perceived behavioral control of information technology: A field theory approach". Journal of Management Information Systems, 28(3), pp. 201-234.

[15] G. Eysenbach. 2000. "Consumer health informatics". British Medical Journal, 320(7251), p. 1713. 
[16] P. N. Finlay, and A. C. Mitchell. 1994. "Perceptions of the benefits from the introduction of CASE: an empirical study”. MIS quarterly, pp. 353-370.

[17] Greenbaum, T. L. 1998. The handbook for focus group research. Sage.

[18] R. Haux. 1997. "Aims and tasks of medical informatics". International journal of medical informatics, 44(1), pp. 9-20.

[19] Z. Jiang, C. S. Heng, and B. C. Choi. 2013. "Privacy concerns and privacy-protective behavior in synchronous online social interactions". Information Systems Research, 24(3), pp. 579-595.

[20] S. Kang, K. H. Lim, M. S. Kim, and H. D. Yang. 2012. „A Multilevel Analysis of the Effect of Group Appropriation on Collaborative Technologies Use and Performance". Information Systems Research, 23(1), pp. 214-230.

[21] R. Koppel, J. P. Metlay, A. Cohen, B. Abaluck, A. R. Localio, S. E. Kimmel, and B. L. Strom. 2005. "Role of computerized physician order entry systems in facilitating medication errors". Jama, 293(10), pp. 1197-1203.

[22] H. Liang, and Y. Xue. 2009. "Avoidance of information technology threats: a theoretical perspective". MIS quarterly, pp. 71-90.

[23] B. Niehaves, and R. Plattfaut. 2014. "Internet adoption by the elderly: employing IS technology acceptance theories for understanding the age-related digital divide". European Journal of Information Systems, 23(6), pp. 708-726.

[24] C. O'hEocha, X. Wang, and K. Conboy. 2012. "The use of focus groups in complex and pressurised IS studies and evaluation using Klein and Myers principles for interpretive research". Information Systems Journal, 22(3), pp. 235-256.

[25] R. Owen, J. Stilgoe, P. Macnaghten, M. Gorman, E. Fisher, and D. Guston. 2013. "A framework for responsible innovation". In Responsible innovation: managing the responsible emergence of science and innovation in society, pp. 27-50.

[26] A. H. Pollack, U. Backonja, A. D. Miller, S. R. Mishra, M. Khelifi, L. Kendall, and W. Pratt. 2016. "Closing the Gap: Supporting Patients' Transition to Self-Management after Hospitalization". In Proceedings of the CHI Conference on Human Factors in Computing Systems, pp. 5324-5336.

[27] Ryan, G. W., and H. R. Bernard. 2000. Data management and analysis methods.

[28] B. Samhan. 2017. "Patients' Resistance towards Health Information Technology A Perspective of the Dual Factor
Model of IT Usage". In Proceedings of the 50th Hawaii International Conference on System Sciences.

[29] J. D. Smeddinck, M. Herrlich, and R. Malaka. 2015. "Exergames for physiotherapy and rehabilitation: a mediumterm situated study of motivational aspects and impact on functional reach". In Proceedings of the ACM Conference on Human Factors in Computing Systems, pp. 4143-4146.

[30] B. C. Stahl, M. C. Tremblay, and C. M. LeRouge. 2011. "Focus groups and critical social IS research: how the choice of method can promote emancipation of respondents and researchers". European Journal of Information Systems, 20(4), pp. 378-394.

[31] B. C. Stahl, G. Eden, and M. Jirotka. 2013. "Responsible research and innovation in information and communication technology: Identifying and engaging with the ethical implications of ICTs". Responsible innovation, pp. 199-218.

[32] B. C. Stahl, G. Eden, M. Jirotka, and M. Coeckelbergh. 2014. „From computer ethics to responsible research and innovation in ICT: The transition of reference discourses informing ethics-related research in information systems". Information and Management, 51(6), pp. 810-818.

[33] J. Stilgoe, R. Owen, and P. Macnaghten. 2013. "Developing a framework for responsible innovation". Research Policy, 42(9), pp. 1568-1580.

[34] R. Tang, X. D. Yang, S. Bateman, J. Jorge, and A. Tang. 2015. "Physio@Home: Exploring visual guidance and feedback techniques for physiotherapy exercises". In Proceedings of the 33rd Annual ACM Conference on Human Factors in Computing Systems, pp. 4123-4132.

[35] Von Kardorff, E., I. Steinke, and U. Flick. (Eds.). 2008. Qualitative Forschung: Ein Handbuch. Rowohlt Taschenbuch-Verlag.

[36] R. Von Schomberg. 2011. „Towards responsible research and innovation in the information and communication technologies and security technologies fields".

[37] R. Von Schomberg. 2013. "A vision of responsible research and innovation". In Responsible innovation: Managing the responsible emergence of science and innovation in society, pp. 51-74.

[38] A. J. Young, L. H. Smith, E. J. Rouse, and L. J. Hargrove. 2014. "A comparison of the real-time controllability of pattern recognition to conventional myoelectric control for discrete and simultaneous movements". Journal of neuroengineering and rehabilitation, 11(1), p. 5. 